\title{
Late seroma of the breast in association with COVID-19 infection: two case reports
}

\author{
Pablo Martínez Núñez ${ }^{1}$ (1) Mónica Pérez González² · Ángel Juárez Cordero ${ }^{1}$
}

Received: 10 August 2021 / Accepted: 7 October 2021 / Published online: 12 November 2021

(c) The Author(s), under exclusive licence to Springer-Verlag GmbH Germany, part of Springer Nature 2021

\begin{abstract}
The SARS-CoV-2 pandemic is giving rise to a wide range of clinical manifestations, which can affect all organs and systems. Due to the enormous and constantly growing number of infected people, even the rarest complications will be observed in a very high number of patients. Late periprosthetic seroma of the breast has gained importance since the description of Breast Implant Associated Anaplastic Large Cell Lymphoma (BIA-ALCL), but this entity is not the only cause. Among the causes of non-BIA-ALCL late seroma, we can include those of mechanical origin and those of non-mechanical origin. Within the latter, the common denominator is the triggering of an aberrant immune reaction that generates an inflammatory exudate from the periprosthetic capsule that gives rise to the seroma. We present two cases of late breast seroma as a manifestation of SARS-CoV-2 infection.
\end{abstract}

Level of evidence: Level V, diagnostic study.

Keywords Late seroma $\cdot$ Breast seroma $\cdot$ COVID-19 $\cdot$ SARS-CoV-2 $\cdot$ BIA-ALCL

\section{Introduction}

Severe acute respiratory syndrome coronavirus 2 (SARSCoV-2) infections have caused over 200 million known cases of coronavirus disease 2019 (COVID-19) worldwide. Although significant knowledge has been acquired about this disease, new clinical manifestations posing diagnostic challenges are frequently reported [1]. We present two cases of late seromas in patients with breast implants that occurred as a complication of COVID-19 infection.

Pablo Martínez Núñez

pablomartineznunez@hotmail.com

$1 \quad$ Plastic Surgery Department, La Zarzuela University Hospital, Comunidad de Madrid, Edificio E, $1^{\text {a }}$ planta, Calle de Pleyades, 25, 28023 Madrid, Spain

2 Radiology and Radiodiagnostic Department, Infanta Cristina University Hospital, Comunidad de Madrid, Parla, Spain

\section{Case reports}

\section{Case 1}

A 44-year-old patient presented to our clinic with a sudden volume increase of her left breast, associated with intense discomfort; she complained of flu-like symptoms for the previous 4 days. The patient had bilateral Mentor Siltex breast prostheses implanted 5 years before. She denied previous trauma. Physical examination revealed a significantly increased volume of her left breast, as well as tightness and redness of the overlying skin. An ultrasound study showed abundant periprosthetic fluid without signs of infection. An ultrasound-guided puncture of the fluid collection yielded $200 \mathrm{cc}$ of serous material (Fig. 1). Cultures were negative; immunohistochemical analysis excluded BIA-ALCL. Two days later, the patient was referred to the Emergency Room for worsening respiratory symptoms; a SARS-CoV-2 COVID-19 PCR test of a nasopharyngeal swab was positive. The patient did not require hospitalization and recovered uneventfully. The seroma of the breast resolved shortly thereafter. 


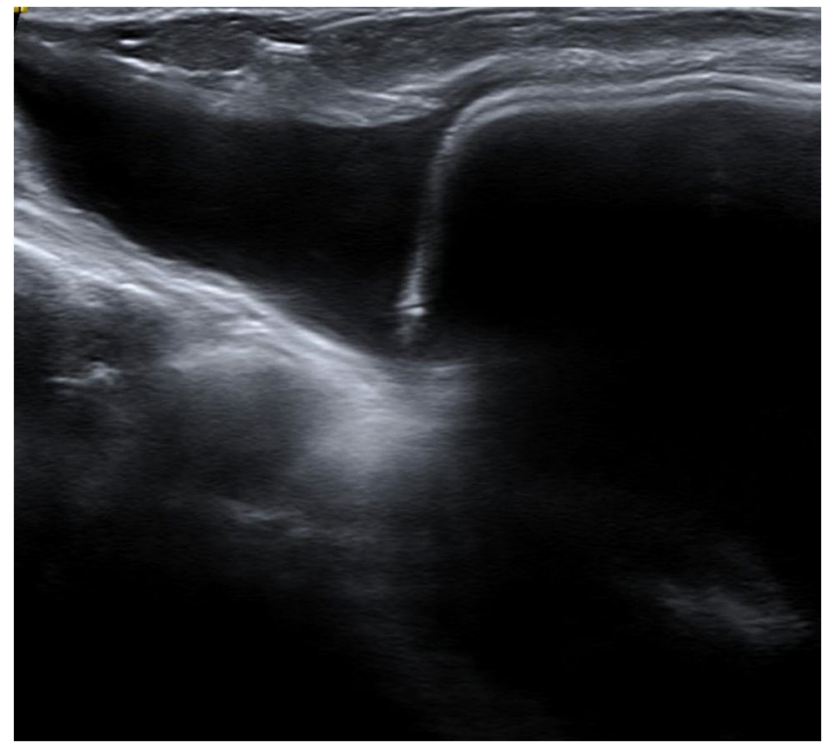

Fig. 1 Discrete amount of anechoic periprosthetic fluid, without septa in its interior in relation to seroma

\section{Case 2}

A 48-year-old patient presented to our clinic with a significant volume increase of her left breast, associated with a feeling of intense pressure at the same location. Eight years before she had been diagnosed with left breast cancer that was treated with mastectomy, followed by breast reconstruction using a 445-cc Allergan 410 implant (Allergan Inc., Irvine, Calif.); her right breast was symmetrized by augmentation with a 185-cc Allergan 410 breast implant. She had an excellent clinical course and achieved full remission. At the time of presentation, she complained of having respiratory symptoms that had developed almost simultaneously to the changes on her breast. A SARS-CoV-2 COVID-19 PCR test of a nasopharyngeal swab was positive, confirming the diagnosis of COVID-19. An ultrasound-guided puncture of the breast yielded $180 \mathrm{cc}$ of periprosthetic serous fluid (Fig. 2). Analysis of the fluid showed an acute inflammatory response with abundant neutrophils; immunohistochemical analysis excluded BIA-ALCL. The seroma recurred while there was worsening of the patient's respiratory symptoms. Finally, once the patient's condition improved, the periprosthetic collection was surgically drained; there were no further relapses.

\section{Discussion}

Late periprosthetic breast seroma appears at least 1 year after prosthesis implantation surgery [2-4]. Before the description of BIA-ALCL, these seromas were associated with a

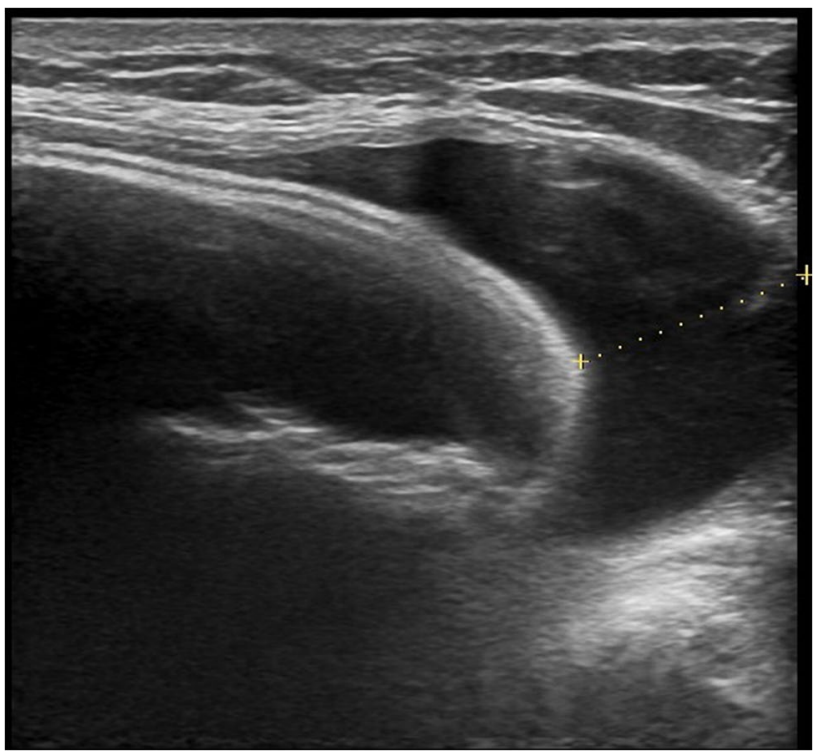

Fig. 2 Left periprosthetic breast seroma

variety of conditions, with many cases lacking a clearly defined cause. Non-BIA-ALCL late seromas are classified as mechanical and non-mechanical, according to their cause. The main hypothesis for mechanical seromas states that friction forces stimulate the production of inflammatory fluid around the prosthesis $[2,3,5,6]$; this occurs almost exclusively in patients with macrotextured implants (97\%), and is commonly located in the submuscular layer $(62 \%)$ $[2,7]$. The macrotextured surface of the prosthesis helps the periprosthetic capsule to adhere to the prosthesis cover like Velcro; however, this union is fragile and can break easily, as it occurs with trauma. The breakage of this adhesion allows rubbing of two surfaces against each other, generating an inflammatory reaction that leads to the accumulation of serous fluid around the prosthesis [2]. A variety of nonmechanical causes for late periprosthetic seromas have been described: allergic reactions to the components of silicone prostheses, clinical and subclinical bacterial infections, viral infections, and prosthesis breakage [8]. These etiologies have in common an immune-mediated inflammatory local reaction, where the release of cytokines, histamine, serotonin, and prostaglandins, together with vasodilation contribute to increase the interstitial fluid and favor the arrival of inflammatory cells; this reaction modifies the osmotic gradient and results in fluid accumulation around the prosthesis $[3,9]$.

Imaging techniques play a leading role in the diagnosis of periprosthetic breast seromas. Ultrasound is considered the diagnostic technique of choice; besides confirming the existence of a periprosthetic collection, it allows its drainage $[4,10,11]$. Magnetic resonance is only used in difficult cases 
when a more precise assessment of the prosthesis status is required or another associated pathology is suspected.

Several reports propose different options for the management of late seromas [4, 12]. Microbiological and cytological analyses are essential to rule out infectious and tumoral etiologies. Once the diagnosis of non-BIAALCL late seroma has been made, the management options described in the literature include ultrasoundguided drainage [7, 13], treatment with antivirals or antibiotics [9], and surgery with prosthesis removal and complete capsulectomy $[5,7,12-14]$. The appropriate treatment should be based on radiological and laboratory findings. According to current algorithms, late breast seromas should be treated initially as infectious; empirical antibiotic treatment should be started after appropriate samples for cultures have been obtained. Definite treatment will vary according to each patient's characteristics. Conservative treatment with further observation may be an option in the absence of infection or tumor. Surgical exploration is indicated for recurrent seromas, and in those with associated suspicious signs. Recommended surgical treatment consists of prosthesis removal and complete capsulectomy, with or without placement of a new implant $[4,12]$.

Clinical manifestations of SARS-CoV-2 infection are multiple and can affect practically any organ and system. In this report, we present 2 cases of late periprosthetic breast seroma that occurred as a complication of COVID-19 infection in patients with breast implants.

Authors' contributions Not applicable.

Funding None

Data Availability Not applicable.

Code availability Not applicable.

\section{Declarations}

Ethics approval All procedures performed in studies involving human participants were in accordance with the ethical standards of the institutional and/or national research committee and with the 1964 Helsinki Declaration and its later amendments or comparable ethical standards. For this kind of study, formal consent from a local ethics committee is not required.

Consent to participate Patients signed informed consent regarding publishing their data and photographs.
Consent for publication The participant has consented to the submission of the case report to the journal.

Conflict of interest Pablo Martínez Núñez, Mónica Pérez González, and Ángel Juárez Cordero declare no conflict of interest.

\section{References}

1. SeyedAlinaghi S, Afsahi AM, MohsseniPour M, Behnezhad F, Salehi MA, Barzegary A, Mirzapour P, Mehraeen E, Dadras O (2021) Late complications of COVID-19; a systematic review of current evidence. Arch Acad Emerg Med 9:e14. https://doi.org/ 10.22037/aaem.v9i1.1058

2. Hall-Findlay EJ (2011) Breast implant complication review: double capsules and late seromas. Plast Reconstr Surg 127:56-66. https://doi.org/10.1097/PRS.0b013e3181fad34d

3. Park BY, Lee DH, Lim SY, Pyon JK, Mun GH, Oh KS, Bang SI (2014) Is late seroma a phenomenon related to textured implants? A report of rare complications and a literature review. Aesthetic Plast Surg 38:139-145. https://doi.org/10.1007/ s00266-013-0232-z

4. Bengtson B, Brody GS, Brown MH, Glicksman C, Hammond D, Kaplan H, Maxwell GP, Oefelein MG, Reisman NR, Spear SL, Jewell ML (2011) Managing late periprosthetic fluid collections (seroma) in patients with breast implants: a consensus panel recommendation and review of the literature. Plast Reconstr Surg 128:1-7. https://doi.org/10.1097/PRS.0b013e318217fdb0

5. Oliveira VM, Roveda Junior D, Lucas FB, Lucarelli AP, Martins MM, Rinaldi JF, Aoki T (2007) Late seroma after breast augmentation with silicone prostheses: a case report. Breast J 13:421-423. https://doi.org/10.1111/j.1524-4741.2007.00453.x

6. Roman S, Perkins D (2005) Progressive spontaneous unilateral enlargement of the breast twenty-two years after prosthetic breast augmentation. Br J Plast Surg 58:88-91. https://doi.org/10.1016/j. bjps.2004.04.002

7. Spear SL, Rottman SJ, Glicksman C, Brown M, Al-Attar A (2012) Late seromas after breast implants: theory and practice. Plast Reconstr Surg 130:423-435. https://doi.org/10.1097/PRS.0b013 e3182589ea9

8. Flassbeck D, Pfleiderer B, Klemens P, Heumann KG, Eltze E, Hirner AV (2003) Determination of siloxanes, silicon, and platinum in tissues of women with silicone gel-filled implants. Anal Bioanal Chem 375:356-362. https://doi.org/10.1007/ s00216-002-1694-z

9. Pinchuk V, Tymofii O (2011) Seroma as a late complication after breast augmentation. Aesthetic Plast Surg 35:303-314. https://doi. org/10.1007/s00266-010-9607-6

10. Graña López L, Vázquez Caruncho M, VillaresArmas A (2016) Management of late seroma in patients with breast implants: the role of the radiologists. Breast J 22:705-707. https://doi.org/10. 1111/tbj. 12665

11. Sylvester-Hvid A, Avnstorp MB, Wagenblast L, Lock-Andersen J, Matzen SH (2017) Case report: breast seroma mimicking breast implants. Int J Surg Case Rep 40:73-76. https://doi.org/10.1016/j. ijscr.2017.08.061

12. Tebbetts JB (2011) Diagnosis and management of seroma following breast augmentation: an update. Plast Reconstr Surg 128:1725. https://doi.org/10.1097/PRS.0b013e3182134aa3 
13 Mazzocchi M, Dessy LA, Corrias F, Scuderi N (2012) A clinical study of late seroma in breast implantation surgery. Aesthetic Plast Surg 36:97-104. https://doi.org/10.1007/ s00266-011-9755-3

14. Roth FS, Gould DJ, Chike-Obi CJ, Bullocks JM (2012) Late seroma during pregnancy, a rare complication in prosthetic breast augmentation: case report. J Plast Reconstr Aesthet Surg 65:973976. https://doi.org/10.1016/j.bjps.2011.11.022

Publisher's Note Springer Nature remains neutral with regard to jurisdictional claims in published maps and institutional affiliations. 